\title{
Article
}

\section{Integral representations for local dilogarithm and trilogarithm functions}

\section{Masato Kobayashi}

Department of Engineering, Kanagawa University, 3-27-1 Rokkaku-bashi, Yokohama 221-8686, Japan.; masato210@gmail.com

Communicated by: Waqas Nazeer

Received: 9 September 2021; Accepted: 16 October 2021; Published: 31 October 2021.

Abstract: We show new integral representations for dilogarithm and trilogarithm functions on the unit interval. As a consequence, we also prove (1) new integral representations for Apéry, Catalan constants and Legendre $\chi$ functions of order 2, 3, (2) a lower bound for the dilogarithm function on the unit interval, (3) new Euler sums.

Keywords: Apéry constant; Catalan constant; Dilogarithm; Euler sum; Inverse sine function; Riemann zeta function; Trilogarithm; Wallis integral.

MSC: Primary: 33E20; Secondary: 11G55; 11M06; 11M41.

\section{Introduction}

\section{Polylogarithm function}

The polylogarithm function

$$
\operatorname{Li}_{s}(z)=\sum_{n=1}^{\infty} \frac{z^{n}}{n^{s}}=z+\frac{z^{2}}{2^{s}}+\frac{z^{3}}{3^{s}}+\cdots, \quad s, z \in \mathbb{C},|z|<1
$$

plays a significant role in many areas of number theory; its origin, the dilogarithm $\mathrm{Li}_{2}(z)$, dates back to Abel, Euler, Kummer, Landen and Spence etc. See Kirillov [1], Lewin [2], Zagier [3] for more details. The main theme of this article is to better understand the relation between the dilogarithm, trilogarithm $\mathrm{Li}_{3}(z)$ functions and zeta values $\zeta(2), \zeta(3)$ (Apéry constant), $\zeta(4)$ in terms of new integral representations.

\section{Main results}

First, we wish to briefly explain work of Boo Rim Choe (1987) [4], Ewell (1990) [5] and Williams-Yue (1993) [6, p.1582-1583] which motivated us. Their common idea is that, from Maclaurin series involving $\sin ^{-1} x$, they each derived certain infinite sums related to $\zeta(2)$ and $\zeta(3)$ with termwise Wallis integral. Figure 1 gives summary of this.

In this article, we reformulate their ideas introducing Wallis operator and naturally extend their results.

- We find new integral representations for $\mathrm{Li}_{2}(t), \mathrm{Li}_{3}(t)$, Legendre $\chi$ functions of order 2,3 and even for Apéry, Catalan constants (Theorems 2, 5, Corollaries 3, 6).

Table 1. Summary of Boo Rim Choe, Ewell and Williams-Yue's work

\begin{tabular}{c|ccc} 
Boo Rim Choe & $\sin ^{-1} x=\sum_{n=0}^{\infty} \frac{(2 n-1) ! !}{(2 n) ! !} \frac{x^{2 n+1}}{2 n+1} \rightarrow \sum_{n=0}^{\infty} \frac{1}{(2 n+1)^{2}}=\frac{3}{4} \zeta(2)=\frac{\pi^{2}}{8}$ \\
\hline Ewell & $\frac{\sin ^{-1} x}{x}=\sum_{n=0}^{\infty} \frac{(2 n-1) ! !}{(2 n) ! !} \frac{x^{2 n}}{2 n+1} \rightarrow \sum_{n=0}^{\infty} \frac{1}{(2 n+1)^{3}}=\frac{7}{8} \zeta(3)$ \\
\hline Williams-Yue & $\frac{\left(\sin ^{-1} x\right)^{2}}{x}=\frac{1}{2} \sum_{n=1}^{\infty} \frac{(2 n) ! !}{(2 n-1) ! !} \frac{x^{2 n-1}}{n^{2}} \rightarrow \frac{\pi}{8} \sum_{n=1}^{\infty} \frac{1}{n^{3}}=\frac{\pi}{8} \zeta(3)$
\end{tabular}


- We give a lower bound for $\mathrm{Li}_{2}(t)$ on the unit interval (Theorem 7).

- Making use of $\left(\sin ^{-1} x\right)^{3}$ and $\left(\sin ^{-1} x\right)^{4}$, we prove new Euler sums (Theorem 8).

\section{Notation}

Throughout this paper, $n$ denotes a nonnegative integer. Let

$$
\begin{aligned}
(2 n) ! ! & =2 n(2 n-2) \cdots 4 \cdot 2, \\
(2 n-1) ! ! & =(2 n-1)(2 n-3) \cdots 3 \cdot 1 .
\end{aligned}
$$

In particular, we understand that $(-1) ! !=0 ! !=1$. Moreover, let

$$
w_{n}=\frac{(n-1) ! !}{n ! !}
$$

Notice the relation $w_{2 n} w_{2 n+1}=\frac{1}{2 n+1}$ as we will see in the sequel.

Remark 1. 1. The sequence $\left\{w_{n}\right\}$ appears in Wallis integral as

$$
\int_{0}^{\pi / 2} \sin ^{n} x= \begin{cases}\frac{\pi}{2} w_{n} & n \text { even } \\ w_{n} & n \text { odd }\end{cases}
$$

2. It also appears in the literature in the disguise of central binomial coefficients as

$$
w_{2 n}=\frac{(2 n-1) ! !}{(2 n) ! !}=\frac{1}{2^{2 n}}\left(\begin{array}{c}
2 n \\
n
\end{array}\right) .
$$

See Apéry [7], van der Poorten [8], for example.

Unless otherwise specified, $t, u, x, y$ are real numbers. By $\sin ^{-1} x$ and $\cos ^{-1} x$, we mean the real inverse sine and $\operatorname{cosine}$ functions $(\arcsin x, \arccos x)$, that is,

$$
\begin{aligned}
& y=\sin ^{-1} x \quad \Longleftrightarrow \quad x=\sin y, \quad-\frac{\pi}{2} \leq y \leq \frac{\pi}{2} \\
& y=\cos ^{-1} x \quad \Longleftrightarrow \quad x=\cos y, \quad 0 \leq y \leq \pi
\end{aligned}
$$

Fact 1 (Gradshteyn-Ryzhik [9, p.60, 61]).

$$
\begin{gathered}
\sin ^{-1} t=\sum_{n=0}^{\infty} w_{2 n} \frac{t^{2 n+1}}{2 n+1}, \quad|t| \leq 1 . \\
\left(\sin ^{-1} t\right)^{2}=\frac{1}{2} \sum_{n=1}^{\infty} \frac{1}{w_{2 n}} \frac{t^{2 n}}{n^{2}}, \quad|t| \leq 1 .
\end{gathered}
$$

Further, $\sinh ^{-1} x=\log \left(x+\sqrt{x^{2}+1}\right)(x \in \mathbb{R})$ denotes the inverse hyperbolic sine function (some authors write $\operatorname{arsinh} x, \operatorname{arcsinh} x$ or $\operatorname{argsinh} x$ for this one).

\section{Dilogarithm function}

\subsection{Definition}

Definition 1. For $0 \leq t \leq 1$, the dilogarithm function is

$$
\operatorname{Li}_{2}(t)=\sum_{n=1}^{\infty} \frac{t^{n}}{n^{2}}
$$

In particular, $\operatorname{Li}_{2}(1)=\zeta(2)=\frac{\pi^{2}}{6}$. 
It is possible to describe its even part by $\mathrm{Li}_{2}$ itself since

$$
\sum_{n=1}^{\infty} \frac{t^{2 n}}{(2 n)^{2}}=\frac{1}{4} \sum_{n=1}^{\infty} \frac{\left(t^{2}\right)^{n}}{n^{2}}=\frac{1}{4} \operatorname{Li}_{2}\left(t^{2}\right) .
$$

Its odd part is called the Legendre $\chi$ function of order 2:

$$
\chi_{2}(t)=\sum_{n=1}^{\infty} \frac{t^{2 n-1}}{(2 n-1)^{2}} .
$$

Here is a fundamental relation of these two parts.

\section{Observation 1.}

$$
\mathrm{Li}_{2}(t)=\chi_{2}(t)+\frac{1}{4} \mathrm{Li}_{2}\left(t^{2}\right)
$$

Definition 2. Define

$$
\operatorname{Ti}_{2}(t)=\sum_{n=1}^{\infty} \frac{(-1)^{n-1}}{(2 n-1)^{2}} t^{2 n-1}
$$

as a signed analog of $\chi_{2}(t)$.

This is also called the inverse tangent integral of order 2 because of the integral representations

$$
\mathrm{Ti}_{2}(t)=\int_{0}^{t} \frac{\tan ^{-1} x}{x} d x
$$

\subsection{Wallis operator}

Let $\mathbb{R}[[t]]$ denote the set of power series in $t$ over real coefficients. Set

$$
F(t)=\{f \in \mathbb{R}[[t]] \mid f(t) \text { is convergent for }|t| \leq 1\} .
$$

Definition 3. For $f \in F(t)$, define $W: F(t) \rightarrow F(t)$ by

$$
W f(t)=\int_{0}^{1} f(t u) \frac{d u}{\sqrt{1-u^{2}}}
$$

Call $W$ the Wallis operator.

Remark 2. [9, p.17] Power series may be integrated and differentiated termwise inside the circle of convergence without changing the radius of convergence. In the sequel, we will frequently use this without mentioning explicitly.

It is now helpful to understand $W$ coefficientwise.

Lemma 1. Let $f(t)=\sum_{n=0}^{\infty} a_{n} t^{n} \in F(t)$. Then

$$
W f(t)=\sum_{n=0}^{\infty} a_{2 n}\left(\frac{\pi}{2} w_{2 n}\right) t^{2 n}+\sum_{n=0}^{\infty} a_{2 n+1} w_{2 n+1} t^{2 n+1}
$$

\section{Proof.}

$$
\begin{aligned}
W f(t) & =\int_{0}^{1} f(t u) \frac{d u}{\sqrt{1-u^{2}}} \\
& =\int_{0}^{1}\left(\sum_{n=0}^{\infty} a_{2 n} t^{2 n} u^{2 n}+\sum_{n=0}^{\infty} a_{2 n+1} t^{2 n+1} u^{2 n+1}\right) \frac{d u}{\sqrt{1-u^{2}}} \\
& =\sum_{n=0}^{\infty} a_{2 n} t^{2 n} \int_{0}^{1} u^{2 n} \frac{d u}{\sqrt{1-u^{2}}}+\sum_{n=0}^{\infty} a_{2 n+1} t^{2 n+1} \int_{0}^{1} u^{2 n+1} \frac{d u}{\sqrt{1-u^{2}}}
\end{aligned}
$$




$$
=\sum_{n=0}^{\infty} a_{2 n}\left(\frac{\pi}{2} w_{2 n}\right) t^{2 n}+\sum_{n=0}^{\infty} a_{2 n+1} w_{2 n+1} t^{2 n+1}
$$

Observe that $W$ is linear in the sense that $W(f+g)=W(f)+W(g)$ and $W(c f)=c W(f)$ for $f, g \in$ $F(t), c \in \mathbb{R}$.

\subsection{Main Theorem 1}

Lemma 2. All of the following are convergent power series for $|t| \leq 1$.

$$
\begin{aligned}
& \sin ^{-1} t=\sum_{n=0}^{\infty} w_{2 n} \frac{t^{2 n+1}}{2 n+1} . \\
& \frac{1}{2}\left(\sin ^{-1} t\right)^{2}=\sum_{n=1}^{\infty} \frac{1}{w_{2 n}} \frac{t^{2 n}}{(2 n)^{2}} . \\
& \sin ^{-1} t+\frac{1}{\pi}\left(\sin ^{-1} t\right)^{2}=\sum_{n=0}^{\infty} w_{2 n} \frac{t^{2 n+1}}{2 n+1}+\sum_{n=1}^{\infty} \frac{2}{\pi w_{2 n}} \frac{t^{2 n}}{(2 n)^{2}} . \\
& \sinh ^{-1} t=\sum_{n=0}^{\infty}(-1)^{n} w_{2 n} \frac{t^{2 n+1}}{2 n+1} . \\
& \frac{1}{2}\left(\sinh ^{-1} t\right)^{2}=\sum_{n=1}^{\infty} \frac{(-1)^{n-1}}{w_{2 n}} \frac{t^{2 n}}{(2 n)^{2}} .
\end{aligned}
$$

Proof. We already saw (3) and (4) in Introduction. (5) is (3) $+\frac{2}{\pi}(4)$. (6) and (7) follow from (3), (4) and $\sinh ^{-1} z=\frac{1}{i} \sin ^{-1}(i z)$ (for all $z \in \mathbb{C}$ ) [9, p.56].

Theorem 2. For $0 \leq t \leq 1$, all of the following hold;

$$
\begin{aligned}
& \chi_{2}(t)=\int_{0}^{1} \frac{\sin ^{-1}(t u)}{\sqrt{1-u^{2}}} d u . \\
& \frac{1}{4} L i_{2}\left(t^{2}\right)=\frac{1}{\pi} \int_{0}^{1} \frac{\left(\sin ^{-1}(t u)\right)^{2}}{\sqrt{1-u^{2}}} d u . \\
& L i_{2}(t)=\int_{0}^{1} \frac{\sin ^{-1}(t u)+\frac{1}{\pi}\left(\sin ^{-1}(t u)\right)^{2}}{\sqrt{1-u^{2}}} d u . \\
& T i_{2}(t)=\int_{0}^{1} \frac{\sinh ^{-1}(t u)}{\sqrt{1-u^{2}}} d u . \\
& \frac{\pi}{2}\left(\frac{1}{4} L i_{2}\left(t^{2}\right)-\frac{1}{8} L i_{2}\left(t^{4}\right)\right)=\int_{0}^{1} \frac{\frac{1}{2}\left(\sinh ^{-1} t u\right)^{2}}{\sqrt{1-u^{2}}} d u .
\end{aligned}
$$

Proof. Note that these are equivalent to the following statements:

$$
\begin{aligned}
& W\left(\sin ^{-1} t\right)=\chi_{2}(t) . \\
& W\left(\frac{1}{2}\left(\sin ^{-1} t\right)^{2}\right)=\frac{\pi}{2} \cdot \frac{1}{4} \operatorname{Li}_{2}\left(t^{2}\right) . \\
& W\left(\sin ^{-1} t+\frac{1}{\pi}\left(\sin ^{-1} t\right)^{2}\right)=\mathrm{Li}_{2}(t) . \\
& W\left(\sinh ^{-1} t\right)=\mathrm{Ti}_{2}(t) . \\
& W\left(\frac{1}{2}\left(\sinh ^{-1} t\right)^{2}\right)=\frac{\pi}{2}\left(\frac{1}{4} \operatorname{Li}_{2}\left(t^{2}\right)-\frac{1}{8} \operatorname{Li}_{2}\left(t^{4}\right)\right) .
\end{aligned}
$$

With Lemmas 1 and 2, we can verify (13)-(16) by checking coefficients of those series. For example,

$$
W\left(\sin ^{-1} t\right)=W\left(\sum_{n=0}^{\infty} w_{2 n} \frac{t^{2 n+1}}{2 n+1}\right)=\sum_{n=0}^{\infty} w_{2 n} w_{2 n+1} \frac{t^{2 n+1}}{2 n+1}=\sum_{n=0}^{\infty} \frac{t^{2 n+1}}{(2 n+1)^{2}}=\chi_{2}(t) .
$$


It remains to show (17).

$$
\begin{aligned}
W\left(\frac{1}{2}\left(\sinh ^{-1} t\right)^{2}\right) & =\sum_{n=1}^{\infty} \frac{(-1)^{n-1}}{w_{2 n}}\left(w_{2 n} \frac{\pi}{2}\right) \frac{t^{2 n}}{(2 n)^{2}} \\
& =\frac{\pi}{2} \sum_{n=1}^{\infty} \frac{(-1)^{n-1}}{(2 n)^{2}} t^{2 n} \\
& =\frac{\pi}{2}\left(\sum_{n=1}^{\infty} \frac{1}{(2 n)^{2}} t^{2 n}-2 \sum_{n=1}^{\infty} \frac{1}{(4 n)^{2}} t^{4 n}\right) \\
& =\frac{\pi}{2}\left(\frac{1}{4} \operatorname{Li}_{2}\left(t^{2}\right)-\frac{1}{8} \operatorname{Li}_{2}\left(t^{4}\right)\right) .
\end{aligned}
$$

\section{Corollary 3.}

$$
\begin{aligned}
& \int_{0}^{1} \frac{\sin ^{-1} u}{\sqrt{1-u^{2}}} d u=\frac{3}{4} \zeta(2)=\frac{\pi^{2}}{8} . \\
& \frac{2}{\pi} \int_{0}^{1} \frac{\frac{1}{2}\left(\sin ^{-1} u\right)^{2}}{\sqrt{1-u^{2}}} d u=\frac{1}{4} \zeta(2)=\frac{\pi^{2}}{24} . \\
& \int_{0}^{1}\left(\sin ^{-1} u+\frac{1}{\pi}\left(\sin ^{-1} u\right)^{2}\right) \frac{d u}{\sqrt{1-u^{2}}}=\zeta(2)=\frac{\pi^{2}}{6} . \\
& \int_{0}^{1} \frac{\sinh ^{-1} u}{\sqrt{1-u^{2}}} d u=G . \\
& \int_{0}^{1} \frac{\frac{1}{2}\left(\sinh ^{-1} u\right)^{2}}{\sqrt{1-u^{2}}} d u=\frac{\pi}{16} \zeta(2)=\frac{\pi^{3}}{96}
\end{aligned}
$$

Proof. These are $\chi_{2}(1), \frac{1}{4} \mathrm{Li}_{2}\left(1^{2}\right), \mathrm{Li}_{2}(1), \mathrm{Ti}_{2}(1)$ and $\frac{\pi}{2}\left(\frac{1}{4} \mathrm{Li}_{2}\left(1^{2}\right)-\frac{1}{8} \mathrm{Li}_{2}\left(1^{4}\right)\right)$.

\section{Trilogarithm function}

\subsection{Definition}

Definition 4. The trilogarithm function for $0 \leq t \leq 1$ is

$$
\mathrm{Li}_{3}(t)=\sum_{n=1}^{\infty} \frac{t^{n}}{n^{3}}
$$

Its odd part is the Legendre $\chi$ function of order 3:

$$
\chi_{3}(t)=\sum_{n=1}^{\infty} \frac{t^{2 n-1}}{(2 n-1)^{3}} .
$$

In particular, $\operatorname{Li}_{3}(1)=\zeta(3)$ and $\chi_{3}(1)=\frac{7}{8} \zeta(3)$.

\section{Observation 4 .}

$$
\operatorname{Li}_{3}(t)=\chi_{3}(t)+\frac{1}{8} \operatorname{Li}_{3}\left(t^{2}\right)
$$

Further, a signed analog of $\chi_{3}(t)$ is

$$
\mathrm{Ti}_{3}(t)=\sum_{n=1}^{\infty} \frac{(-1)^{n-1}}{(2 n-1)^{3}} t^{2 n-1}
$$




\subsection{Main Theorem 2}

\section{Lemma 3.}

$$
\begin{aligned}
& \int_{0}^{t} \frac{\sin ^{-1} y}{y} d y=\sum_{n=0}^{\infty} w_{2 n} \frac{t^{2 n+1}}{(2 n+1)^{2}} . \\
& \int_{0}^{t} \frac{\frac{1}{2}\left(\sin ^{-1} y\right)^{2}}{y} d y=\sum_{n=1}^{\infty} \frac{1}{w_{2 n}} \frac{t^{2 n}}{(2 n)^{3}} . \\
& \int_{0}^{t} \frac{\sin ^{-1} y+\frac{1}{\pi}\left(\sin ^{-1} y\right)^{2}}{y} d y=\sum_{n=0}^{\infty} w_{2 n} \frac{t^{2 n+1}}{(2 n+1)^{2}}+\sum_{n=1}^{\infty} \frac{2}{\pi w_{2 n}} \frac{t^{2 n}}{(2 n)^{3}} . \\
& \int_{0}^{t} \frac{\sinh ^{-1} y}{y} d y=\sum_{n=0}^{\infty}(-1)^{n} w_{2 n} \frac{t^{2 n+1}}{(2 n+1)^{2}} . \\
& \int_{0}^{t} \frac{\frac{1}{2}\left(\sinh ^{-1} y\right)^{2}}{y} d y=\sum_{n=1}^{\infty} \frac{(-1)^{n-1}}{w_{2 n}} \frac{t^{2 n}}{(2 n)^{3}} .
\end{aligned}
$$

Proof. We can derive all of these by integrating (3)-(7) termwise.

As a consequence, we obtain the equalities below (cf. (13)-(17)).

$$
\begin{aligned}
& W\left(\int_{0}^{t} \frac{\sin ^{-1} y}{y} d y\right)=\chi_{3}(t) . \\
& W\left(\int_{0}^{t} \frac{\frac{1}{2}\left(\sin ^{-1} y\right)^{2}}{y} d y\right)=\frac{\pi}{2} \cdot \frac{1}{8} \operatorname{Li}_{3}\left(t^{2}\right) . \\
& W\left(\int_{0}^{t} \frac{\sin ^{-1} y+\frac{1}{\pi}\left(\sin ^{-1} y\right)^{2}}{y} d y\right)=\mathrm{Li}_{3}(t) . \\
& W\left(\int_{0}^{t} \frac{\sinh ^{-1} y}{y} d y\right)=\mathrm{Ti}_{3}(t) \text {. } \\
& W\left(\int_{0}^{t} \frac{\frac{1}{2}\left(\sinh ^{-1} y\right)^{2}}{y} d y\right)=\sum_{n=1}^{\infty} \frac{(-1)^{n-1}}{w_{2 n}}\left(w_{2 n} \frac{\pi}{2}\right) \frac{t^{2 n}}{(2 n)^{3}} \\
& =\frac{\pi}{2} \sum_{n=1}^{\infty} \frac{(-1)^{n-1}}{(2 n)^{3}} t^{2 n} \\
& =\frac{\pi}{2}\left(\sum_{n=1}^{\infty} \frac{1}{(2 n)^{3}} t^{2 n}-2 \sum_{n=1}^{\infty} \frac{1}{(4 n)^{3}} t^{4 n}\right) \\
& =\frac{\pi}{2}\left(\frac{1}{8} \operatorname{Li}_{3}\left(t^{2}\right)-\frac{1}{32} \operatorname{Li}_{3}\left(t^{4}\right)\right) \text {. }
\end{aligned}
$$

In this way, the five functions above come to possess double integral representations. For example,

$$
\chi_{3}(t)=\int_{0}^{1}\left(\int_{0}^{t u} \frac{\sin ^{-1} y}{y} d y\right) \frac{d u}{\sqrt{1-u^{2}}}
$$

We can indeed simplify such integrals to single ones by exchanging order of integrals.

\section{Theorem 5.}

$$
\begin{aligned}
& \chi_{3}(t)=\int_{0}^{1} \frac{\sin ^{-1}(t x) \cos ^{-1} x}{x} d x . \\
& \frac{1}{8} L i_{3}\left(t^{2}\right)=\frac{2}{\pi} \int_{0}^{1} \frac{\frac{1}{2}\left(\sin ^{-1}(t x)\right)^{2} \cos ^{-1} x}{x} d x \\
& L i_{3}(t)=\int_{0}^{1} \frac{\left(\sin ^{-1}(t x)+\frac{1}{\pi}\left(\sin ^{-1}(t x)\right)^{2}\right) \cos ^{-1} x}{x} d x
\end{aligned}
$$




$$
\begin{aligned}
& \operatorname{Ti}_{3}(t)=\int_{0}^{1} \frac{\sinh ^{-1}(t x) \cos ^{-1} x}{x} d x . \\
& \frac{\pi}{2}\left(\frac{1}{8} L i_{3}\left(t^{2}\right)-\frac{1}{32} L i_{3}\left(t^{4}\right)\right)=\int_{0}^{1} \frac{\frac{1}{2}\left(\sinh ^{-1}(t x)\right)^{2} \cos ^{-1} x}{x} d x .
\end{aligned}
$$

Proof. We give a proof altogether. For $t=0$, all the equalities hold as $0=0$. Suppose $0<t \leq 1$. Let

$$
f(y) \in\left\{\sin ^{-1} y, \frac{1}{\pi}\left(\sin ^{-1} y\right)^{2}, \sin ^{-1} y+\frac{1}{\pi}\left(\sin ^{-1} y\right)^{2}, \sinh ^{-1} y, \frac{1}{2}\left(\sinh ^{-1} y\right)^{2}\right\} .
$$

Then

$$
\begin{aligned}
W\left(\int_{0}^{t} \frac{f(y)}{y} d y\right) & =\int_{0}^{1} \int_{0}^{t u} \frac{f(y)}{y} d y \frac{d u}{\sqrt{1-u^{2}}} \\
& =\int_{0}^{t} \int_{y / t}^{1} \frac{f(y)}{y} \frac{1}{\sqrt{1-u^{2}}} d u d y \\
& =\int_{0}^{t} \frac{f(y)}{y} \cos ^{-1} \frac{y}{t} d y \\
& =\int_{0}^{1} \frac{f(t x)}{x} \cos ^{-1} x d x
\end{aligned}
$$

\section{Corollary 6.}

$$
\begin{aligned}
& \int_{0}^{1} \frac{\sin ^{-1} x \cos ^{-1} x}{x} d x=\frac{7}{8} \zeta(3) . \\
& \frac{2}{\pi} \int_{0}^{1} \frac{\frac{1}{2}\left(\sin ^{-1} x\right)^{2} \cos ^{-1} x}{x} d x=\frac{1}{8} \zeta(3) . \\
& \int_{0}^{1} \frac{\left(\sin ^{-1} x+\frac{1}{\pi}\left(\sin ^{-1} x\right)^{2}\right) \cos ^{-1} x}{x} d x=\zeta(3) . \\
& \int_{0}^{1} \frac{\sinh ^{-1} x \cos ^{-1} x}{x} d x=\frac{\pi^{3}}{32} . \\
& \int_{0}^{1} \frac{\frac{1}{2}\left(\sinh ^{-1} x\right)^{2} \cos ^{-1} x}{x} d x=\frac{3 \pi}{64} \zeta(3) .
\end{aligned}
$$

Proof. These are $\chi_{3}(1), \frac{1}{8} \mathrm{Li}_{3}\left(1^{2}\right), \mathrm{Li}_{3}(1), \mathrm{Ti}_{3}(1)$ and $\frac{\pi}{2}\left(\frac{1}{8} \mathrm{Li}_{3}\left(1^{2}\right)-\frac{1}{32} \mathrm{Li}_{3}\left(1^{4}\right)\right)$.

\section{Applications}

\subsection{Inequalities}

It is easy to see from the definitions $\operatorname{Li}_{2}(t)=\sum_{n=1}^{\infty} \frac{t^{n}}{n^{2}}$ and $\chi_{2}(t)=\sum_{n=1}^{\infty} \frac{t^{2 n-1}}{(2 n-1)^{2}}(0 \leq t \leq 1)$ that

$$
0 \leq \operatorname{Li}_{2}(t) \leq \frac{\pi^{2}}{6} \quad \text { and } \quad 0 \leq \chi_{2}(t) \leq \frac{\pi^{2}}{8} .
$$

In fact, we can improve these inequalities a little more. For upper bounds, it is immediate that

$$
\begin{aligned}
& \mathrm{Li}_{2}(t)=\sum_{n=1}^{\infty} \frac{t^{n}}{n^{2}} \leq \sum_{n=1}^{\infty} \frac{t}{n^{2}}=\frac{\pi^{2}}{6} t \\
& \chi_{2}(t)=\sum_{n=1}^{\infty} \frac{t^{2 n-1}}{(2 n-1)^{2}} \leq \sum_{n=1}^{\infty} \frac{t}{(2 n-1)^{2}}=\frac{\pi^{2}}{8} t .
\end{aligned}
$$

We next prove nontrivial lower bounds for these functions and also $\mathrm{Ti}_{2}(t)$. 
Theorem 7. For $0 \leq t \leq 1$,

$$
\begin{aligned}
L i_{2}(t) & \geq \frac{4}{3 \pi} \frac{\left(\sin ^{-1} t\right)^{3}}{t} \\
\chi_{2}(t) & \geq \frac{\left(\sin ^{-1} t\right)^{2}}{2 t} \\
\operatorname{Ti}_{2}(t) & \geq \frac{\left(\sinh ^{-1} t\right)^{2}}{2 t} .
\end{aligned}
$$

Before the proof, we need a lemma. It provides another integral representation of $\operatorname{Li}_{2}(t)$ which seems interesting itself.

Lemma 4. For $0 \leq t \leq 1$,

$$
L i_{2}(t)=\frac{8 \sqrt{t}}{\pi} \int_{0}^{1} \frac{\sin ^{-1}(\sqrt{t} x) \cos ^{-1} x}{\sqrt{1-t x^{2}}} d x .
$$

(cf. $\quad \operatorname{Li}_{3}(t)=\frac{8}{\pi} \int_{0}^{1} \frac{\left(\sin ^{-1}(\sqrt{t} x)\right)^{2} \cos ^{-1} x}{x} d x, \quad t \mapsto \sqrt{t}$ in (34).)

Proof. If $t=0$, then both sides are 0 . For $0<t \leq 1$,

$$
\begin{aligned}
\text { RHS } & =\frac{8}{\pi} \int_{0}^{\sqrt{t}} \frac{\sin ^{-1} y}{\sqrt{1-y^{2}}} \cos ^{-1} \frac{y}{\sqrt{t}} d y \\
& =\frac{8}{\pi} \int_{0}^{\sqrt{t}} \frac{\sin ^{-1} y}{\sqrt{1-y^{2}}} \int_{y / \sqrt{t}}^{1} \frac{1}{\sqrt{1-u^{2}}} d u d y \\
& =\frac{8}{\pi} \int_{0}^{1} \int_{0}^{\sqrt{t u}} \frac{\sin ^{-1} y}{\sqrt{1-y^{2}}} d y \frac{1}{\sqrt{1-u^{2}}} d u \\
& =\frac{8}{\pi} W\left(\int_{0}^{\sqrt{t}} \frac{\sin ^{-1} y}{\sqrt{1-y^{2}}} d y\right) \\
& =\frac{8}{\pi} W\left(\frac{1}{2}\left(\sin ^{-1} \sqrt{t}\right)^{2}\right) \\
& =\frac{8}{\pi}\left(\frac{\pi}{2} \frac{1}{4} \operatorname{Li}_{2}\left((\sqrt{t})^{2}\right)\right)=\operatorname{Li}_{2}(t) .
\end{aligned}
$$

Proof of Theorem 7. If $t=0$, then all of (43)-(45) hold as $0 \geq 0$. Suppose $t>0$. Since $\sin ^{-1}$ is increasing on $[0,1], \sin ^{-1}(t x) \leq \sin ^{-1}(\sqrt{t} x)$ for all $0<t, x \leq 1$. Then

$$
\begin{aligned}
& \mathrm{Li}_{2}(t)=\frac{8 \sqrt{t}}{\pi} \int_{0}^{1} \frac{\sin ^{-1}(\sqrt{t} x)}{\sqrt{1-t x^{2}}} \cos ^{-1} x d x \\
& \geq \frac{8 t}{\pi} \int_{0}^{1} \frac{\sin ^{-1}(t x)}{\sqrt{1-t^{2} x^{2}}} \cos ^{-1} x d x \\
& =\frac{8 t}{\pi} \int_{0}^{1} \frac{1}{t}\left(\frac{1}{2}\left(\sin ^{-1} t x\right)^{2}\right) \cos ^{-1} x d x \\
& =\frac{8}{\pi}(\underbrace{\left[\frac{1}{2}\left(\sin ^{-1} t x\right)^{2} \cos ^{-1} x\right]_{0}^{1}}_{0}-\int_{0}^{1} \frac{1}{2}\left(\sin ^{-1} t x\right)^{2} \frac{-1}{\sqrt{1-x^{2}}} d x) \\
& \geq \frac{4}{\pi} \int_{0}^{1} \frac{\left(\sin ^{-1} t x\right)^{2}}{\sqrt{1-t^{2} x^{2}}} d x \\
& =\frac{4}{\pi}\left[\frac{1}{3 t}\left(\sin ^{-1} t x\right)^{3}\right]_{0}^{1}=\frac{4}{3 \pi} \frac{\left(\sin ^{-1} t\right)^{3}}{t} .
\end{aligned}
$$


Next, we prove (44). Note that

$$
\frac{\sin ^{-1}(t x)}{\sqrt{1-t^{2} x^{2}}} \leq \frac{\sin ^{-1}(t x)}{\sqrt{1-x^{2}}}
$$

for $0<t, x<1$. Integrate these from 0 to 1 in $x$ so that

$$
\begin{aligned}
\int_{0}^{1} \frac{\sin ^{-1}(t x)}{\sqrt{1-t^{2} x^{2}}} d x & \leq \int_{0}^{1} \frac{\sin ^{-1}(t x)}{\sqrt{1-x^{2}}} d x, \\
{\left[\frac{\left(\sin ^{-1}(t x)\right)^{2}}{2 t}\right]_{0}^{1} } & \leq \chi_{2}(t), \\
\frac{\left(\sin ^{-1} t\right)^{2}}{2 t} & \leq \chi_{2}(t) .
\end{aligned}
$$

Quite similarly, for $0<t, x<1$, it also holds that

$$
\begin{aligned}
\frac{\sinh ^{-1}(t x)}{\sqrt{1+t^{2} x^{2}}} & \leq \frac{\sinh ^{-1}(t x)}{\sqrt{1-x^{2}}} \\
\int_{0}^{1} \frac{\sinh ^{-1}(t x)}{\sqrt{1+t^{2} x^{2}}} d x & \leq \int_{0}^{1} \frac{\sinh ^{-1}(t x)}{\sqrt{1-x^{2}}} d x=\mathrm{Ti}_{2}(t) .
\end{aligned}
$$

The left hand side is

$$
\left[\frac{\sinh ^{-1}(t x)^{2}}{2 t}\right]_{0}^{1}=\frac{\left(\sinh ^{-1} t\right)^{2}}{2 t}
$$

\subsection{Euler sums}

Definition 5. A harmonic number is $H_{n}=\sum_{k=1}^{n} \frac{1}{k}$. More generally, for $m, n \geq 1$, an $(m, n)$-harmonic number is

$$
H_{n}^{(m)}=\sum_{k=1}^{n} \frac{1}{k^{m}}
$$

In particular, $H_{n}^{(1)}=H_{n}$. Any series involving such numbers is called an Euler sum.

Vălean [10, p.292-293] presents truly remarkable Euler sums such as

$$
\begin{aligned}
\sum_{n=1}^{\infty} \frac{H_{n}^{2}}{n^{2}} & =\frac{17}{4} \zeta(4) \\
\sum_{n=1}^{\infty} \frac{H_{n}^{2}}{n^{3}} & =\frac{7}{2} \zeta(5)-\zeta(2) \zeta(3) \\
\sum_{n=1}^{\infty} \frac{H_{n}^{2}}{n^{4}} & =\frac{97}{24} \zeta(6)-2 \zeta^{2}(3) \\
\sum_{n=1}^{\infty} \frac{H_{n}^{2}}{n^{5}} & =6 \zeta(7)-\zeta(2) \zeta(5)-\frac{5}{2} \zeta(3) \zeta(4), \\
\sum_{n=1}^{\infty} \frac{H_{n} H_{n}^{(2)}}{n^{2}} & =\zeta(2) \zeta(3)+\zeta(5) .
\end{aligned}
$$

There are many ideas to prove such formulas; Borwein and Bradley [11] gives thirty two proofs for

$$
\sum_{n=1}^{\infty} \frac{H_{n-1}}{n^{2}}=\zeta(3)=8 \sum_{n=1}^{\infty} \frac{(-1)^{n} H_{n-1}}{n^{2}}
$$


by integrals, polylogarithm functions, Fourier series and hypergeometric functions etc. Here, as an application of our main idea, Wallis operators, we prove two new Euler sums. Let

$$
O_{n}^{(2)}=H_{2 n-1}^{(2)}-\frac{1}{4} H_{n-1}^{(2)}=\sum_{k=1}^{2 n-1} \frac{1}{k^{2}}-\sum_{k=1}^{n-1} \frac{1}{(2 k)^{2}}=\sum_{k=0}^{n-1} \frac{1}{(2 k+1)^{2}} .
$$

\section{Theorem 8.}

$$
\begin{aligned}
& \sum_{n=0}^{\infty} \frac{O_{n}^{(2)}}{(2 n+1)^{2}}=\frac{\pi^{4}}{384}=\frac{15}{64} \zeta(4) . \\
& \sum_{n=1}^{\infty} \frac{H_{n-1}^{(2)}=\frac{\pi^{4}}{120}=\frac{3}{4} \zeta(4) .}{n^{2}}
\end{aligned}
$$

For the proof, we make use of less-known Maclaurin series for $\left(\sin ^{-1} t\right)^{3}$ and $\left(\sin ^{-1} t\right)^{4}$; thus we can interpret this result as a natural subsequence of Boo, Ewell and Williams-Yue's work.

\section{Lemma 5.}

$$
\begin{aligned}
&\left(\sin ^{-1} t\right)^{3}=\sum_{n=0}^{\infty}\left(6 O_{n}^{(2)}\right) w_{2 n} \frac{t^{2 n+1}}{2 n+1} . \\
&\left(\sin ^{-1} t\right)^{4}=\frac{1}{2} \sum_{n=1}^{\infty}\left(3 H_{n-1}^{(2)}\right) \frac{1}{w_{2 n}} \frac{t^{2 n}}{n^{2}} . \\
&\text { cf. } \left.\quad \sin ^{-1} t=\sum_{n=0}^{\infty} w_{2 n} \frac{t^{2 n+1}}{2 n+1}, \quad\left(\sin ^{-1} t\right)^{2}=\frac{1}{2} \sum_{n=1}^{\infty} \frac{1}{w_{2 n}} \frac{t^{2 n}}{n^{2}}\right) .
\end{aligned}
$$

Proof. First, write $\left(\sin ^{-1} t\right)^{3}=\sum_{n=0}^{\infty} A_{n} t^{2 n+1}, A_{n} \in \mathbb{R}$ and let $a_{n}=\frac{2 n+1}{w_{2 n}} A_{n}(n \geq 0)$. It is enough to show that $a_{n}=6 O_{n}^{(2)}$. Since the series $\left(\sin ^{-1} t\right)^{3}=\left(t+\frac{t^{3}}{6}+\cdots\right)^{3}$ starts from the $t^{3}$ term, $A_{0}=a_{0}=0$. For convenience, set

$$
f_{n}(x)=\frac{\sin ^{2 n+1} x}{(2 n+1) !}
$$

Then

$$
\begin{gathered}
f_{n}^{\prime}(x)=\frac{\sin ^{2 n} x}{(2 n) !} \cos x \\
f_{n}^{\prime \prime}(x)=\frac{1}{(2 n) !}\left(2 n \sin ^{2 n-1} x\left(1-\sin ^{2} x\right)-\sin ^{2 n+1} x\right)=f_{n-1}(x)-(2 n+1)^{2} f_{n}(x) .
\end{gathered}
$$

Now let $x=\sin ^{-1} t\left(-\frac{\pi}{2} \leq x \leq \frac{\pi}{2}\right), b_{n}=(2 n-1) !$ !. Recall that

$$
\sin ^{-1} t=\sum_{n=0}^{\infty} w_{2 n} \frac{t^{2 n+1}}{2 n+1}
$$

In terms of $x, b_{n}, f_{n}(x)$, this is

$$
x=\sum_{n=0}^{\infty} w_{2 n} \frac{\sin ^{2 n+1} x}{2 n+1}=\sum_{n=0}^{\infty} \frac{(2 n-1) ! !}{(2 n) ! !}(2 n) ! \frac{\sin ^{2 n+1} x}{(2 n+1) !}=\sum_{n=0}^{\infty} b_{n}^{2} f_{n}(x) .
$$

Thus,

$$
x^{3}=\sum_{n=0}^{\infty} A_{n} \sin ^{2 n+1} x=\sum_{n=0}^{\infty} a_{n}\left(w_{2 n} \frac{\sin ^{2 n+1} x}{2 n+1}\right)=\sum_{n=0}^{\infty} a_{n} b_{n}^{2} f_{n}(x) .
$$


Differentiate both sides twice in $x$ :

$$
\begin{aligned}
6 x & =\sum_{n=0}^{\infty} a_{n} b_{n}^{2} f_{n}^{\prime \prime}(x) \\
& =\sum_{n=0}^{\infty} a_{n} b_{n}^{2}\left(f_{n-1}(x)-(2 n+1)^{2} f_{n}(x)\right) \\
& =\sum_{n=0}^{\infty}\left(a_{n+1} b_{n+1}^{2} f_{n}(x)-a_{n} b_{n}^{2}(2 n+1)^{2} f_{n}(x)\right), \\
6 \sum_{n=0}^{\infty} b_{n}^{2} f_{n}(x) & =\sum_{n=0}^{\infty}\left(a_{n+1} b_{n+1}^{2} f_{n}(x)-a_{n} b_{n}^{2}(2 n+1)^{2} f_{n}(x)\right) .
\end{aligned}
$$

Equating coefficients of $f_{n}(x)$ yields

$$
6 b_{n}^{2}=a_{n+1} b_{n+1}^{2}-a_{n} b_{n}^{2}(2 n+1)^{2}, \quad n \geq 0 .
$$

Since $b_{n+1}=(2 n+1) b_{n}$ and $b_{n} \neq 0$, we must have

$$
a_{n+1}-a_{n}=\frac{6}{(2 n+1)^{2}} .
$$

With $a_{0}=0$, we now arrive at

$$
a_{n}=\sum_{k=0}^{n-1} \frac{6}{(2 k+1)^{2}}=6 O_{n}^{(2)}
$$

as required.

The proof for (50) proceeds along the same line. Write $\left(\sin ^{-1} t\right)^{4}=\frac{1}{2} \sum_{n=0}^{\infty} C_{n} t^{2 n}, C_{n} \in \mathbb{R}$ and let $c_{n}=$ $C_{n} w_{2 n} n^{2}(n \geq 1)$. It is enough to show that $c_{n}=3 H_{n-1}^{(2)}$. Since the series $\left(\sin ^{-1} t\right)^{4}$ starts from the $t^{4}$ term, $C_{1}=c_{1}=0$. For convenience, set

$$
g_{n}(x)=\frac{\sin ^{2 n} x}{(2 n) !}
$$

Then

$$
\begin{gathered}
g_{n}^{\prime}(x)=\frac{\sin ^{2 n-1} x}{(2 n-1) !} \cos x, \\
g_{n}^{\prime \prime}(x)=\frac{1}{(2 n-1) !}\left((2 n-1) \sin ^{2 n-2} x\left(1-\sin ^{2} x\right)-\sin ^{2 n} x\right)=g_{n-1}(x)-(2 n)^{2} g_{n}(x) .
\end{gathered}
$$

Now let $x=\sin ^{-1} t\left(-\frac{\pi}{2} \leq x \leq \frac{\pi}{2}\right), d_{n}=2^{n}(n-1) !$. Recall that

$$
\left(\sin ^{-1} t\right)^{2}=\frac{1}{2} \sum_{n=1}^{\infty} \frac{1}{w_{2 n}} \frac{t^{2 n}}{n^{2}} .
$$

In terms of $x, d_{n}, g_{n}(x)$, this is

$$
x^{2}=\frac{1}{2} \sum_{n=1}^{\infty} \frac{1}{w_{2 n}} \frac{\sin ^{2 n} x}{n^{2}}=\frac{1}{2} \sum_{n=1}^{\infty} \frac{(2 n) ! !}{(2 n-1) ! !} \frac{(2 n) !}{n^{2}} \frac{\sin ^{2 n} x}{(2 n) !}=\frac{1}{2} \sum_{n=1}^{\infty} d_{n}^{2} g_{n}(x) .
$$

Thus,

$$
x^{4}=\frac{1}{2} \sum_{n=1}^{\infty} c_{n}\left(\frac{1}{w_{2 n}} \frac{\sin ^{2 n} x}{n^{2}}\right)=\frac{1}{2} \sum_{n=1}^{\infty} c_{n} d_{n}^{2} g_{n}(x) .
$$

Differentiate both sides twice in $x$ :

$$
\begin{aligned}
12 x^{2} & =\frac{1}{2} \sum_{n=1}^{\infty} c_{n} d_{n}^{2} g_{n}^{\prime \prime}(x) \\
& =\frac{1}{2} \sum_{n=1}^{\infty} c_{n} d_{n}^{2}\left(g_{n-1}(x)-(2 n)^{2} g_{n}(x)\right)
\end{aligned}
$$




$$
\begin{aligned}
& =\frac{1}{2} \sum_{n=0}^{\infty} c_{n+1} d_{n+1}^{2} g_{n}(x)-\frac{1}{2} \sum_{n=1}^{\infty} c_{n} d_{n}^{2}(2 n)^{2} g_{n}(x) \\
& =\frac{1}{2} \sum_{n=1}^{\infty} c_{n+1} d_{n+1}^{2} g_{n}(x)-\frac{1}{2} \sum_{n=1}^{\infty} c_{n} d_{n}^{2}(2 n)^{2} g_{n}(x) \quad\left(c_{1}=0\right) \\
& =\frac{1}{2} \sum_{n=1}^{\infty}\left(c_{n+1} d_{n+1}^{2}-c_{n} d_{n}^{2}(2 n)^{2}\right) g_{n}(x), \\
\frac{12}{2} \sum_{n=1}^{\infty} d_{n}^{2} g_{n}(x) & =\frac{1}{2} \sum_{n=1}^{\infty}\left(c_{n+1} d_{n+1}^{2}-c_{n} d_{n}^{2}(2 n)^{2}\right) g_{n}(x) .
\end{aligned}
$$

Equating coefficients of $g_{n}(x)$ yields

$$
\frac{12}{2} d_{n}^{2}=\frac{1}{2}\left(c_{n+1} d_{n+1}^{2}-c_{n} d_{n}^{2}(2 n)^{2}\right), \quad n \geq 1 .
$$

Since $d_{n+1}=2 n d_{n}$ and $d_{n} \neq 0$, we must have

$$
c_{n+1}-c_{n}=\frac{12}{(2 n)^{2}} .
$$

With $c_{1}=0$, we conclude that

$$
c_{n}=\sum_{k=1}^{n-1} \frac{12}{(2 k)^{2}}=\sum_{k=1}^{n-1} \frac{3}{k^{2}}=3 H_{n-1}^{(2)} .
$$

Proof of Theorem 8. Note that

$$
\begin{aligned}
W\left(\frac{1}{6}\left(\sin ^{-1} t\right)^{3}\right) & =\sum_{n=1}^{\infty} \frac{\widetilde{O}_{n}^{(2)} w_{2 n}}{2 n+1} w_{2 n+1} t^{2 n+1} \\
& =\sum_{n=1}^{\infty} \frac{\widetilde{O}_{n}^{(2)}}{(2 n+1)^{2}} t^{2 n+1}
\end{aligned}
$$

Clearly, $t=1$ gives the sum for (47). Therefore,

$$
\left.W\left(\frac{1}{6}\left(\sin ^{-1} t\right)^{3}\right)\right|_{t=1}=\int_{0}^{1} \frac{1}{6}\left(\sin ^{-1} u\right)^{3} \frac{d u}{\sqrt{1-u^{2}}}=\left[\frac{1}{24}\left(\sin ^{-1} u\right)^{4}\right]_{0}^{1}=\frac{\pi^{4}}{384} .
$$

Similarly, we have

$$
W\left(\frac{2}{3}\left(\sin ^{-1} t\right)^{4}\right)=\frac{\pi}{2} \sum_{n=1}^{\infty} \frac{H_{n-1}^{(2)}}{n^{2}} t^{2 n}
$$

so that

$$
\left.W\left(\frac{2}{3}\left(\sin ^{-1} t\right)^{4}\right)\right|_{t=1}=\int_{0}^{1} \frac{2}{3}\left(\sin ^{-1} u\right)^{4} \frac{d u}{\sqrt{1-u^{2}}}=\left[\frac{2}{15}\left(\sin ^{-1} u\right)^{5}\right]_{0}^{1}=\frac{\pi^{5}}{240} .
$$

We conclude that

$$
\sum_{n=1}^{\infty} \frac{H_{n-1}^{(2)}}{n^{2}}=\frac{2}{\pi}\left(\frac{\pi^{5}}{240}\right)=\frac{\pi^{4}}{120}
$$

Remark 3. 1. (47) is a variation of De Doelder's formula $\sum_{n=1}^{\infty} \frac{O_{n}^{(2)}}{n^{2}}=\frac{\pi^{4}}{32}$ [12, p.1196 (13)] and (48) gives another proof of $\sum_{n=1}^{\infty} \frac{H_{n}^{(2)}}{n^{2}}=\frac{7}{4} \zeta(4)[10$, p.286] because

$$
\sum_{n=1}^{\infty} \frac{H_{n}^{(2)}}{n^{2}}=\sum_{n=1}^{\infty}\left(\frac{H_{n-1}^{(2)}}{n^{2}}+\frac{1}{n^{4}}\right)=\frac{3}{4} \zeta(4)+\zeta(4)=\frac{7}{4} \zeta(4) .
$$


2. After preparation of the manuscript, Christophie Vignat kindly told me that recently Guo-Lim-Qi (2021) [13] described Maclaurin series of integer powers of arcsin. In fact, it was the result from J.M. Borwein-Chamberland (2007) [14].

\subsection{Integral evaluation}

As byproduct of our discussions, we find evaluation of many integrals with known special values of $\mathrm{Li}_{2}(t), \mathrm{Li}_{3}(t)$. Here, we record several examples. Let $\phi=\frac{1+\sqrt{5}}{2}$ be the golden ratio. Observe that

$$
\phi^{-1}=\frac{\sqrt{5}-1}{2}, \quad \phi^{-2}=\frac{3-\sqrt{5}}{2} .
$$

We write $\log ^{2} x$ for $(\log x)^{2}$. Note that

$$
\log ^{2}\left(\phi^{-1}\right)=\left(\log \left(\phi^{-1}\right)\right)^{2}=(-\log (\phi))^{2}=(\log (\phi))^{2}=\log ^{2}(\phi) .
$$

Fact 2 ([2]).

$$
\begin{aligned}
\operatorname{Li}_{2}\left(\phi^{-1}\right) & =-\log ^{2}(\phi)+\frac{\pi^{2}}{10} . \\
\operatorname{Li}_{2}\left(\phi^{-2}\right) & =-\log ^{2}(\phi)+\frac{\pi^{2}}{15} . \\
\operatorname{Li}_{3}\left(\phi^{-2}\right) & =\frac{4}{5} \zeta(3)-\frac{2 \pi^{2}}{15} \log \phi+\frac{2}{3} \log ^{3} \phi . \\
\operatorname{Li}_{2}\left(\frac{1}{2}\right) & =\frac{\pi^{2}}{12}-\frac{1}{2} \log ^{2} 2 . \\
\operatorname{Li}_{3}\left(\frac{1}{2}\right) & =\frac{7}{8} \zeta(3)-\frac{\pi^{2}}{12} \log 2+\frac{1}{6} \log ^{3} 2 .
\end{aligned}
$$

\section{Corollary 9.}

$$
\begin{aligned}
& \int_{0}^{1} \frac{\sin ^{-1}\left(\phi^{-1} u\right)}{\sqrt{1-u^{2}}} d u=-\frac{3}{4} \log ^{2}(\phi)+\frac{\pi^{2}}{12} \\
& \int_{0}^{1} \frac{\frac{1}{2}\left(\sin ^{-1} \frac{u}{\sqrt{2}}\right)^{2}}{\sqrt{1-u^{2}}} d u=\frac{\pi}{8}\left(\frac{\pi^{2}}{12}-\frac{1}{2} \log ^{2} 2\right) . \\
& \frac{16}{\pi} \int_{0}^{1} \frac{1}{2}\left(\sin ^{-1} \phi^{-1} x\right)^{2} \frac{\cos ^{-1} x}{x} d x=\frac{4}{5} \zeta(3)-\frac{2 \pi^{2}}{15} \log \phi+\frac{2}{3} \log ^{3} \phi \\
& \int_{0}^{1} \frac{1}{2}\left(\sinh ^{-1} \phi^{-1 / 2} u\right)^{2} \frac{d u}{\sqrt{1-u^{2}}}=\frac{\pi}{2}\left(-\frac{1}{8} \log ^{2} \phi+\frac{\pi^{2}}{60}\right) . \\
& \frac{16}{\pi} \int_{0}^{1} \frac{1}{2}\left(\sin ^{-1} \frac{x}{\sqrt{2}}\right)^{2} \frac{\cos ^{-1} x}{x} d x=\frac{7}{8} \zeta(3)-\frac{\pi^{2}}{12} \log 2+\frac{1}{6} \log ^{3} 2 .
\end{aligned}
$$

\section{Proof.}

$$
\begin{aligned}
& \int_{0}^{1} \frac{\sin ^{-1}\left(\phi^{-1} u\right)}{\sqrt{1-u^{2}}} d u=\chi_{2}\left(\phi^{-1}\right)=\operatorname{Li}_{2}\left(\phi^{-1}\right)-\frac{1}{4} \operatorname{Li}_{2}\left(\phi^{-2}\right) \\
&=\left(-\log ^{2}(\phi)+\frac{\pi^{2}}{10}\right)-\frac{1}{4}\left(-\log ^{2}(\phi)+\frac{\pi^{2}}{15}\right)=-\frac{3}{4} \log ^{2}(\phi)+\frac{\pi^{2}}{12} . \\
&\left.W\left(\frac{1}{2}\left(\sin ^{-1} t\right)^{2}\right)\right|_{t=1 / \sqrt{2}}=\frac{\pi}{8} \operatorname{Li}_{2}\left(\frac{1}{2}\right)=\frac{\pi}{8}\left(\frac{\pi^{2}}{12}-\frac{1}{2} \log ^{2} 2\right) .
\end{aligned}
$$

(34) for $t=\phi^{-1}$ with (53) gives (58).

$$
\left.W\left(\frac{1}{2}\left(\sinh ^{-1} t\right)^{2}\right)\right|_{t=\phi^{-1 / 2}}=\frac{\pi}{2}\left(\frac{1}{4} \operatorname{Li}_{2}\left(\phi^{-1}\right)-\frac{1}{8} \operatorname{Li}_{2}\left(\phi^{-2}\right)\right)=\frac{\pi}{2}\left(-\frac{1}{8} \log ^{2} \phi+\frac{\pi^{2}}{60}\right) .
$$


Finally, (34) for $t=1 / \sqrt{2}$ with (55) gives (60).

\section{Concluding remarks}

Here, we record several remarks for our future research.

1. For $0 \leq \alpha \leq 1$, define a generalized Wallis operator

$$
W_{\alpha} f(t)=\int_{0}^{\alpha} f(t u) \frac{d u}{\sqrt{1-u^{2}}}
$$

so that we can deal with more general integrals. Study $W_{\alpha}$, particularly for $\alpha=1 / 2, \sqrt{2} / 2, \sqrt{3} / 2$.

2. Can we show any inequality for $\operatorname{Li}_{3}(t), \chi_{3}(t)$ and $\operatorname{Ti}_{3}(t)$ in a similar way?

3. Discuss $\left(\sinh ^{-1} t\right)^{3},\left(\sinh ^{-1} t\right)^{4}$ and related Euler sums.

4. Wolfram alpha [15] says that

$$
\begin{aligned}
& \int_{0}^{1} \frac{\left(\sin ^{-1} x\right)^{3}}{x} d x=\int_{0}^{\pi / 2} u^{3} \cot u d u=\frac{\pi^{3}}{8} \log 2-\frac{9}{16} \pi \zeta(3), \\
& \int_{0}^{1} \frac{\left(\sin ^{-1} x\right)^{4}}{x} d x=\int_{0}^{\pi / 2} u^{4} \cot u d u=\frac{1}{32}\left(-18 \pi^{2} \zeta(3)+93 \zeta(5)+2 \pi^{4} \log 2\right) .
\end{aligned}
$$

It should be possible to describe such integrals as certain infinite sums with or without numbers $w_{2 n}$. We plan to study those details in subsequent publication.

5. It is interesting that (38) happens to be quite similar to

$$
\int_{0}^{1} \frac{\tan ^{-1} x \cot ^{-1} x}{x} d x=\frac{7}{8} \zeta(3) .
$$

Not often this result appears in this form in the literature, though. Now, let us see how we evaluate this integral. Let

$$
\begin{aligned}
& I=\int_{0}^{1} \frac{\tan ^{-1} x \cot ^{-1} x}{x} d x, \\
& I_{1}=\int_{0}^{1} \frac{\tan ^{-1} x}{x} d x \\
& I_{2}=\int_{0}^{1} \frac{\left(\tan ^{-1} x\right)^{2}}{x} d x .
\end{aligned}
$$

Then

$$
\begin{aligned}
I & =\int_{0}^{1} \frac{\tan ^{-1} x \cot ^{-1} x}{x} d x \\
& =\int_{0}^{1} \frac{\tan ^{-1} x\left(\frac{\pi}{2}-\tan ^{-1} x\right)}{x} d x=\frac{\pi}{2} I_{1}-I_{2} .
\end{aligned}
$$

We can compute $I_{1}$ and $I_{2}$ as follows.

$$
\begin{aligned}
I_{1} & =\int_{0}^{1} \frac{\tan ^{-1} x}{x} d x=\int_{0}^{1} \sum_{n=0}^{\infty} \frac{(-1)^{n}}{2 n+1} x^{2 n} d x \\
& =\sum_{n=0}^{\infty} \frac{(-1)^{n}}{2 n+1} \int_{0}^{1} x^{2 n} d x=\sum_{n=0}^{\infty} \frac{(-1)^{n}}{(2 n+1)^{2}}=G .
\end{aligned}
$$

For $I_{2}$, recall from Fourier analysis that

$$
\log \left(\tan \frac{y}{2}\right)=-2 \sum_{n=0}^{\infty} \frac{1}{2 n+1} \cos (2 n+1) y, \quad 0<y<\pi .
$$


It follows that

$$
\begin{aligned}
I_{2} & =\int_{0}^{1} \frac{\left(\tan ^{-1} x\right)^{2}}{x} d x=\left[y=2 \tan ^{-1} x\right] \frac{1}{4} \int_{0}^{\pi / 2} \frac{y^{2}}{\sin y} d y \\
& =\frac{1}{4}\left(\left[y^{2} \log \left(\tan \frac{y}{2}\right)\right]_{0}^{\pi / 2}-\int_{0}^{\pi / 2} 2 y \log \left(\tan \frac{y}{2}\right) d y\right) \\
& =-\frac{1}{2} \int_{0}^{\pi / 2} y\left(-2 \sum_{n=0}^{\infty} \frac{1}{2 n+1} \cos (2 n+1) y\right) d y \\
& =\sum_{n=0}^{\infty} \frac{1}{2 n+1} \int_{0}^{\pi / 2} y \cos (2 n+1) y d y \\
& =\sum_{n=0}^{\infty} \frac{1}{2 n+1}\left(\left[y \frac{\sin (2 n+1) y}{2 n+1}\right]_{0}^{\pi / 2}-\int_{0}^{\pi / 2} \frac{\sin (2 n+1) y}{2 n+1} d y\right) \\
& =\sum_{n=0}^{\infty}\left(\frac{\pi}{2} \frac{(-1)^{n}}{(2 n+1)^{2}}-\frac{1}{(2 n+1)^{3}}\right)=\frac{\pi G}{2}-\frac{7}{8} \zeta(3) .
\end{aligned}
$$

Finally, we see

$$
I=\frac{\pi G}{2}-\left(\frac{\pi G}{2}-\frac{7}{8} \zeta(3)\right)=\frac{7}{8} \zeta(3)
$$

Open Question What if we replace $\tan ^{-1}$ by $\tanh ^{-1}$ ?

In this article, we encountered many integral representations for dilogarithm, trilogarithm and hence $\zeta(2)$, the Catalan constant $G$ and $\zeta(3)$ as a reformulation of Boo Rim Choe (1987) [4], Ewell (1990) [5] and Williams-Yue (1993) [6] on the inverse sine function. As an application, we also proved new Euler sums. Indeed, there are subsequent results on multiple zeta and $t$-values $\zeta(3,2, \cdots, 2), t(3,2, \cdots, 2)$ as Hoffman and Zagier discussed in [16,17]. We will write them with more details at another opportunity.

Conflicts of Interest: "The author declares no conflict of interest".

\section{References}

[1] Kirillov, A. N. (1995). Dilogarithm identities. Progress of Theoretical Physics Supplement, 118, 61-142.

[2] Lewin, L. (1981). Polylogarithms and Associated Functions. North-Holland, Amsterdam.

[3] Zagier, D. (2007). The dilogarithm function. In Frontiers in number theory, physics, and geometry II (pp. 3-65). Springer, Berlin, Heidelberg.

[4] Choe, B. R. (1987). An elementary proof of $\sum_{n=1}^{\infty} \frac{1}{n^{2}}=\frac{\pi^{2}}{6}$. The American Mathematical Monthly, 94(7), 662-663.

[5] Ewell, J. A. (1990). A new series representation for $\zeta(3)$. The American Mathematical Monthly, 97(3), 219-220.

[6] Yue, Z. N., \& Williams, K. S. (1993). Some series representations of $\zeta(2 n+1)$. The Rocky Mountain Journal of Mathematics, 23(4), 1581-1592.

[7] Apéry, R. (1979). Irrationalité de $\zeta(2)$ et $\zeta(3)$. Astérisque, 61, 11-13.

[8] van der Poorten, A. (2005). A proof that Euler missed, Apéry's proof of the irrationality of $\zeta(3)$, an informal report. ALF's reprints, Paper, 45, 1-16.

[9] Gradshteyn, I. S., \& Ryzhik, I. M. (2014). Table of Integrals, Series, and Products. Academic press.

[10] Vălean, C. I. (2019). (Almost) Impossible Integrals, Sums, and Series, Problem Books in Mathematics. Springer International Publishing.

[11] Borwein, J. M., \& Bradley, D. M. (2006). Thirty-two Goldbach variations. International Journal of Number Theory, 2(1), 65-103.

[12] Borwein, D., \& Borwein, J. M. (1995). On an intriguing integral and some series related to $\zeta(4)$. Proceedings of the American Mathematical Society, 123(4), 1191-1198.

[13] Guo, B. N., Lim, D., \& Qi, F. (2021). Maclaurin series expansions for powers of inverse (hyperbolic) sine, for powers of inverse (hyperbolic) tangent, and for incomplete gamma functions, with applications to second kind Bell polynomials and generalized logsine function. AIMS Mathematics 6(7), 7494-7517.

[14] Borwein, J. M., \& Chamberland, M. (2007). Integer powers of Arcsin. International Journal of Mathematics and Mathematical Sciences, 2007, Article ID 19381, 10 pages.

[15] Wolfram alpha. https://www.wolframalpha.com.

[16] Hoffman, M. E. (2019). An odd variant of multiple zeta values. Communications in Number Theory and Physics, 13(3), 529-567. 
[17] Zagier, D. (2012). Evaluation of the multiple zeta values $\zeta(2, \cdots, 2,3,2, \cdots, 2)$. Annals of Mathematics, 175, 977-1000.

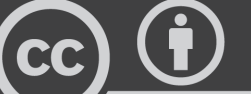

BY
(C) 2021 by the authors; licensee PSRP, Lahore, Pakistan. This article is an open access article distributed under the terms and conditions of the Creative Commons Attribution (CC-BY) license (http://creativecommons.org/licenses/by/4.0/). 